\title{
O-minimal fields with standard part map
}

by

\author{
Jana Maříková (Macomb, IL)
}

\begin{abstract}
Let $R$ be an o-minimal field and $V$ a proper convex subring with residue field $\boldsymbol{k}$ and standard part (residue) map st: $V \rightarrow \boldsymbol{k}$. Let $\boldsymbol{k}_{\text {ind }}$ be the expansion of $\boldsymbol{k}$ by the standard parts of the definable relations in $R$. We investigate the definable sets in $\boldsymbol{k}_{\text {ind }}$ and conditions on $(R, V)$ which imply o-minimality of $\boldsymbol{k}_{\text {ind }}$. We also show that if $R$ is $\omega$-saturated and $V$ is the convex hull of $\mathbb{Q}$ in $R$, then the sets definable in $\boldsymbol{k}_{\text {ind }}$ are exactly the standard parts of the sets definable in $(R, V)$.
\end{abstract}

1. Introduction. Throughout, $R$ is an o-minimal field, that is, an ominimal expansion of a real closed field, and $V$ is a proper convex subring with maximal ideal $\mathfrak{m}$, ordered residue field $\boldsymbol{k}=V / \mathfrak{m}$, and standard part (residue) map st: $V \rightarrow \boldsymbol{k}$. This map induces a map st: $V^{n} \rightarrow \boldsymbol{k}^{n}$ and for $X \subseteq R^{n}$ we put st $X:=\operatorname{st}\left(X \cap V^{n}\right)$. By $\boldsymbol{k}_{\text {ind }}$ we denote the ordered field $\boldsymbol{k}$ expanded by the relations st $X$ with $X \in \operatorname{Def}^{n}(R), n=1,2, \ldots$ Unless indicated otherwise, by "definable" we mean "definable with parameters in the structure $R$ ".

The most important case of a convex subring of $R$ is the convex hull

$$
\mathcal{O}:=\left\{x \in R:|x| \leq q \text { for some } q \in \mathbb{Q}^{>0}\right\}
$$

of $\mathbb{Q}$ in $R$. If $V=\mathcal{O}$, then the ordered field $\boldsymbol{k}$ is archimedean and we identify $\boldsymbol{k}$ with its image in the ordered field $\mathbb{R}$ of real numbers via the unique ordered field embedding of $\boldsymbol{k}$ into $\mathbb{R}$. In particular, if $R$ is $\omega$-saturated and $V=\mathcal{O}$, then $\boldsymbol{k}=\mathbb{R}$.

We consider the following questions:

(1) Under what conditions on $(R, V)$ is $\boldsymbol{k}_{\text {ind }}$ o-minimal?

(2) How complicated are the definable relations in $\boldsymbol{k}_{\text {ind }}$ in terms of the basic relations st $X$ with definable $X \subseteq R^{n}$ ?

Here is a brief history of these problems. In 1983, Cherlin and Dickmann [3] proved quantifier elimination for real closed fields with a proper convex 
subring. In 1995 van den Dries and Lewenberg [7] identified the notion of T-convex subring of an o-minimal field as a suitable analogue of convex subring of a real closed field (here $T$ is the theory of the given o-minimal field). A convex subring $V$ of $R$ is said to be $\operatorname{Th}(R)$-convex if $f(V) \subseteq V$ for every continuous $\emptyset$-definable function $f: R \rightarrow R$. The situation when $V$ is a $\mathrm{Th}(R)$-convex subring of $R$ is well-understood; see [7] and [5]. In particular, $\boldsymbol{k}_{\text {ind }}$ is o-minimal in that case.

The structure $\boldsymbol{k}_{\text {ind }}$ is not always o-minimal, as the example on page 128 shows. However, $\boldsymbol{k}_{\text {ind }}$ is always weakly o-minimal: By a theorem of Baizhanov in [2] (see also [1]), $(R, V)$ is weakly o-minimal, and by an argument just as in the proof of Lemma 4.1, every $Y \subseteq \boldsymbol{k}$ definable in $\boldsymbol{k}_{\text {ind }}$ equals st $X$ for some $X \subseteq V$ definable in $(R, V)$. Hrushovski, Peterzil and Pillay observe in [11] that if $R$ is sufficiently saturated and $V=\mathcal{O}$, then $\boldsymbol{k}_{\text {ind }}$ is o-minimal, because then $\boldsymbol{k}=\mathbb{R}$ and for expansions of the ordered field $\mathbb{R}$ weak ominimality is the same as o-minimality. However, [11] gives no information about question (2) in that situation, which includes cases where $\mathcal{O}$ is not $\operatorname{Th}(R)$-convex; we say more about this in the remark on page 117 .

Good cell decomposition. In [14] we answered (2) for the situation in [11] by means of good cell decomposition, which also gives the o-minimality of $\mathbb{R}_{\text {ind }}$ without using [2]. In the present paper we obtain good cell decomposition (and thus o-minimality of $\boldsymbol{k}_{\text {ind }}$ ) under more general first-order assumptions on the pair $(R, V)$. More precisely, suppose $(R, V) \models \Sigma_{\mathrm{i}}$ where $\Sigma_{\mathrm{i}}$ is defined below. Theorem 2.21 says that then the subsets of $\boldsymbol{k}^{n}$ definable in $\boldsymbol{k}_{\text {ind }}$ are the finite unions of differences st $X \backslash$ st $Y$, where $X, Y \subseteq R^{n}$ are definable. It follows that $\boldsymbol{k}_{\text {ind }}$ is o-minimal. Theorem 2.21 is proved in the same way as the corresponding theorem in [14], except that uses of saturation in [14] are replaced by uses of $\Sigma_{\mathrm{i}}$. Also the proof of Lemma 4.1 in [14] does not generalize to our setting, and this is replaced here by a more elementary proof of Lemma 2.4 below.

The following conditions on $(R, V)$ are related to good cell decomposition. To state these, let $I:=\{x \in R:|x| \leq 1\}$, and for $X \subseteq R^{1+n}$ and $r \in R$, put

$$
X(r):=\left\{x \in R^{n}:(r, x) \in X\right\} .
$$

We let $\mathfrak{m}^{>r}:=\{x \in \mathfrak{m}: x>r\}$ for $r \in \mathfrak{m}$. We define the conditions $\mathcal{I}, \Sigma_{\mathrm{i}}$, $\Sigma_{\mathrm{d}}, \Sigma$, and $\mathcal{C}$ on pairs $(R, V)$ as follows:

$(\mathcal{I})$ if $X, Y \subseteq I^{n}$ are definable, then there is a definable $Z \subseteq I^{n}$ such that st $X \cap$ st $Y=$ st $Z$;

$\left(\Sigma_{\mathrm{i}}\right)$ if $X \subseteq I^{1+n}$ is definable and $X(r) \subseteq X(s)$ for all $r, s \in I$ with $r \leq s$, then there is $\epsilon_{0} \in \mathfrak{m}^{>0}$ such that st $X\left(\epsilon_{0}\right)=$ st $X(\epsilon)$ for all $\epsilon \in \mathfrak{m}^{>\epsilon_{0}}$ 
$\left(\Sigma_{\mathrm{d}}\right)$ if $X \subseteq I^{1+n}$ is definable and $X(r) \supseteq X(s)$ for all $r, s \in I$ with $r \leq s$, then there is $\epsilon_{0} \in \mathfrak{m}^{>0}$ such that st $X\left(\epsilon_{0}\right)=$ st $X(\epsilon)$ for all $\epsilon \in \mathfrak{m}^{>\epsilon_{0}}$

$(\Sigma)$ if $X \subseteq I^{1+n}$ is definable, then there is $\epsilon_{0} \in \mathfrak{m}^{>0}$ such that st $X\left(\epsilon_{0}\right)=$ st $X(\epsilon)$ for all $\epsilon \in \mathfrak{m}^{>\epsilon_{0}}$;

$(\mathcal{C})$ the $\boldsymbol{k}_{\text {ind }}$-definable closed subsets of $\boldsymbol{k}^{n}$ are exactly the sets st $X$ with definable $X \subseteq R^{n}$.

One should add here "for all $n$ and $X, Y$ " as initial clause to $\mathcal{I}$, and likewise with the other conditions. In Section 3 we prove that for all $(R, V)$,

a) $\mathcal{I} \Leftrightarrow \Sigma_{i}$;

b) $\Sigma_{\mathrm{i}} \Rightarrow \boldsymbol{k}_{\text {ind }}$ is o-minimal;

c) $\Sigma \Rightarrow \mathcal{C}$.

In a subsequent paper with van den Dries [8] we shall prove the converse of c), and also $\Sigma_{\mathrm{i}} \Longrightarrow \mathcal{C}$, yielding $\Sigma_{\mathrm{i}} \Leftrightarrow \Sigma$. More recently, the second author shows in [15] the converse of b), so $\Sigma_{\mathrm{i}}$ really yields a first-order axiomatization of the structures $(R, V)$ with o-minimal $\boldsymbol{k}_{\text {ind }}$.

Our definition of $\mathcal{I}$ is not of first-order nature, but by a) it is equivalent to first-order conditions. Similarly $\mathcal{C}$ will turn out to be equivalent to first order conditions by c) and its converse in 8 .

In Section 3 we also show that $(R, V)$ satisfies $\Sigma$ if any of the following holds:

(i) $\operatorname{cofinality}(\mathfrak{m})>2^{|\boldsymbol{k}|}$;

(ii) $V$ is $T$-convex, where $T:=\operatorname{Th}(R)$;

(iii) $R$ is $\omega$-saturated and $V=\mathcal{O}$.

Traces. Call a set $X \subseteq R^{n}$ a trace if $X=Y \cap R^{n}$ for some definable $n$ ary relation $Y$ in some elementary extension of $R$, where we allow parameters from that elementary extension to define $Y$. In Section 4 we assume that $R$ is $\omega$-saturated and $V=\mathcal{O}$, and under these assumptions we characterize the definable sets in $\mathbb{R}_{\text {ind }}$ in terms of traces. As a corollary we show that if $R$ is $\omega$-saturated and $V=\mathcal{O}$, then

$$
\operatorname{Def}^{n}\left(\mathbb{R}_{\text {ind }}\right)=\left\{\text { st } X: X \in \operatorname{Def}^{n}(R, \mathcal{O})\right\} .
$$

We do not know if the analogue of this corollary holds under the more general first-order assumption $\Sigma$. We do know that if $V$ is $\operatorname{Th}(R)$-convex, then, for all $n$,

$$
\operatorname{Def}^{n}\left(\boldsymbol{k}_{\text {ind }}\right)=\left\{\text { st } X: X \in \operatorname{Def}^{n}(R, V)\right\} .
$$

REMARK. In 1996 van den Dries [4] asked the following question: Let $L$ be a language extending the language of ordered rings, and let $T(L, \mathbb{R})$ be the set of all sentences true in all $L$-expansions of the real field. Call $R$ pseudo-real if $R \models T(L, \mathbb{R})$. Is every o-minimal field pseudo-real? 
If $R$ has an archimedean model, then $R$ is pseudo-real, but the converse fails. Consider for example a proper elementary extension of the real field and extend its language by a name for an element $\lambda>\mathbb{R}$. Then the theory of $R$ in the extended language does not have an archimedean model but $R$ is of course pseudo-real as a structure for this extended language.

In 2006 Lipshitz and Robinson [12] considered the ordered Hahn field $\mathbb{R}\left(\left(t^{\mathbb{Q}}\right)\right)$ with operations given by overconvergent power series, and they proved its o-minimality. In 2007 Hrushovski and Peterzil [10] showed that this Lipshitz-Robinson field is not pseudo-real. It is easy to see that if $R$ is a model of the theory $T$ of the Lipshitz-Robinson field, then $\mathcal{O} \subseteq R$ is not $T$-convex.

Preliminaries. We assume familiarity with o-minimal structures and their basic properties; see for example [6]. Throughout, we let $m, n$ range over the set $\mathbb{N}=\{0,1,2, \ldots\}$ of natural numbers. Given a one-sorted structure $\mathcal{M}=(M ; \cdots)$ we let $\operatorname{Def}^{n}(\mathcal{M})$ be the boolean algebra of definable subsets of $M^{n}$. Let $K$ be an ordered field. For $x \in K$ we put $|x|:=\max \{x,-x\}$, for $a=\left(a_{1}, \ldots, a_{n}\right) \in K^{n}$ we put

$$
|a|:=\max \left\{\left|a_{i}\right|: i=1, \ldots, n\right\} \quad \text { if } n>0, \quad|a|:=0 \quad \text { if } n=0,
$$

and for $a, b \in K^{n}$ we put $d(a, b):=|a-b|$. A box in $K^{n}$ is a cartesian product of open intervals

$$
\left(a_{1}-\delta, a_{1}+\delta\right) \times \cdots \times\left(a_{n}-\delta, a_{n}+\delta\right),
$$

where $a=\left(a_{1}, \ldots, a_{n}\right) \in K^{n}$ and $\delta \in K^{>0}$. A $V$-box in $R^{n}$ is a box in $R^{n}$ as above where $a \in V^{n}$ and $\delta \in V^{>\mathfrak{m}}$. So if $B \subseteq R^{n}$ is a $V$-box, then $B \subseteq V^{n}$ and st $B$ contains a box in $\boldsymbol{k}^{n}$.

An interval is always a nonempty open interval $(a, b)$ in $R$, or in $\mathbb{R}$, or in $\boldsymbol{k}$, as specified. We already defined $I:=\{x \in R:|x| \leq 1\}$ and more generally, for each ordered field $K$ we put $I(K):=\{x \in K:|x| \leq 1\}$. For $a \in R^{n}$ and definable nonempty $X \subseteq R^{n}$ we set

$$
d(a, X):=\inf \{d(a, x): x \in X\},
$$

and likewise for $a \in \boldsymbol{k}^{n}$ and definable nonempty $X \subseteq \boldsymbol{k}^{n}$ when $\boldsymbol{k}_{\text {ind }}$ is o-minimal. A set $X \subseteq R^{n}$ is said to be $V$-bounded if there is $a \in V^{>0}$ such that $|x| \leq a$ for all $x \in X$. (For $V=\mathcal{O}$ this is the same as strongly bounded.) The hull of $X \subseteq \boldsymbol{k}^{n}$ is the set $X^{h}:=\mathrm{st}^{-1}(X) \subseteq V^{n}$.

Given sets $X, Y$ and $S \subseteq X \times Y$ we put

$$
S(x):=\{y \in Y:(x, y) \in S\} .
$$

If $X$ is a subset of an ambient set $M$ that is understood from the context, then

$$
X^{c}:=\{x \in M: x \notin X\} .
$$


We often use the following projection maps for $m \leq n$ :

$$
\begin{aligned}
p_{m}^{n}: R^{n} \rightarrow R^{m}, & \left(x_{1}, \ldots, x_{n}\right) \mapsto\left(x_{1}, \ldots, x_{m}\right), \\
\pi_{m}^{n}: \boldsymbol{k}^{n} \rightarrow \boldsymbol{k}^{m}, & \left(x_{1}, \ldots, x_{n}\right) \mapsto\left(x_{1}, \ldots, x_{m}\right) .
\end{aligned}
$$

Given a map $f: X \rightarrow Y$ we let

$$
\Gamma f:=\{(x, y) \in X \times Y: f(x)=y\}
$$

denote its graph.

\section{Good cell decomposition}

2.1. General facts on standard part sets. Recall that $R$ is an ominimal field and $V$ is a proper convex subring of $R$. We begin with some results requiring no extra assumption on $(R, V)$. A very useful fact of this kind is the $V$-box lemma (Corollary 2.5).

Lemma 2.1. If $X \subseteq R^{n}$ is definable, then st $X$ is closed.

Proof. Let $X \subseteq R^{n}$ be definable and assume towards a contradiction that we have an $a \in \operatorname{cl}(\operatorname{st} X) \backslash$ st $X$. Take $a^{\prime} \in R^{n}$ such that st $a^{\prime}=a$. Then, by o-minimality of $R, d\left(a^{\prime}, X\right)$ exists in $R$ and $d\left(a^{\prime}, X\right)>\mathfrak{m}$. So there is a neighborhood $U \subseteq \boldsymbol{k}^{n}$ of $a$ with $U \cap$ st $X=\emptyset$, a contradiction.

Let $\mathrm{St}_{n}$ be the collection of all sets st $X$ with definable $X \subseteq R^{n}$. Note that if $X, Y \in \mathrm{St}_{n}$, then $X \cup Y \in \mathrm{St}_{n}$; if $X \in \mathrm{St}_{m}$ and $Y \in \mathrm{St}_{n}$, then $X \times Y \in \mathrm{St}_{m+n}$. The next lemma is almost obvious. To state it we use the projection maps $\pi=\pi_{m}^{m+n}: \boldsymbol{k}^{m+n} \rightarrow \boldsymbol{k}^{m}$ and $p=p_{m}^{m+n}: R^{m+n} \rightarrow R^{m}$.

Lemma 2.2. Let $X \in \mathrm{St}_{m+n}$. Then

(1) if $X$ is bounded, then $\pi(X) \in \mathrm{St}_{m}$;

(2) if $X=\operatorname{st} X^{\prime}$ where the set $X^{\prime} \subseteq R^{m+n}$ is definable in $R$ and satisfies $X^{\prime} \cap p^{-1}\left(V^{m}\right) \subseteq V^{m+n}$, then $\pi(X) \in \mathrm{St}_{m}$.

Lemma 2.3. If $X \subseteq R$ is definable, then $\mathrm{st} X$ is a finite union of intervals and points in $\boldsymbol{k}$.

Proof. This is immediate from the o-minimality of $R$.

Below, $p$ is the projection map $R^{n+1} \rightarrow R^{n}$ given by $p\left(x_{1}, \ldots, x_{n+1}\right)=$ $\left(x_{1}, \ldots, x_{n}\right)$.

LEMMA 2.4.

$\left(A_{n}\right)$ If $D \subseteq V^{n+1}$ is a $V$-box, and $f: Y \rightarrow R$, where $Y \subseteq V^{n}$, is definable and continuous with $f(Y) \subseteq V$, then there is a $V$-box $B \subseteq D$ with $B \cap \Gamma f=\emptyset$.

$\left(B_{n}\right)$ If $D \subseteq V^{n}$ is a $V$-box, and $\mathcal{C}$ is a decomposition of $D$, then there is $C \in \mathcal{C}$ such that $C$ contains a $V$-box. 
Proof. It is clear that $\left(B_{1}\right)$ holds. We first show that $\left(B_{n}\right)$ implies $\left(A_{n}\right)$. Let $f: Y \rightarrow V$ be definable and continuous, with $Y \subseteq V^{n}$, and let

$$
D=\left(a_{1}, b_{1}\right) \times \cdots \times\left(a_{n+1}, b_{n+1}\right) \subseteq V^{n+1}
$$

be a $V$-box. Take $p, q \in V$ such that $a_{n+1}<p<q<b_{n+1}$ and

$$
q-p, p-a_{n+1}, b_{n+1}-q>\mathfrak{m},
$$

and pick $\delta>\mathfrak{m}$ with $\delta<\min \left\{p-a_{n+1},(q-p) / 2, b_{n+1}-q\right\}$. Define

$$
\begin{aligned}
& X(p):=\left\{x \in p_{n}^{n+1} D \cap Y: f(x) \in(p-\delta, p+\delta)\right\}, \\
& X(q):=\left\{x \in p_{n}^{n+1} D \cap Y: f(x) \in(q-\delta, q+\delta)\right\},
\end{aligned}
$$

and note that $X(p) \cap X(q)=\emptyset$. Take a decomposition $\mathcal{C}$ of $R^{n}$ such that $\mathcal{C}$ partitions the sets $p_{n}^{n+1} D, X(p)$, and $X(q)$. By $\left(B_{n}\right)$, there is $C \in \mathcal{C}$ such that $C \subseteq p_{n}^{n+1} D$ and $C$ contains a $V$-box $P$. Then $P \times(p-\delta, p+\delta)$ or $P \times(q-\delta, q+\delta)$ yields the desired $V$-box $B$.

Next, we show that $\left(A_{n}\right)$ and $\left(B_{n}\right)$ imply $\left(B_{n+1}\right)$. Let $D \subseteq V^{n+1}$ be a $V$-box and let $\mathcal{C}$ be a decomposition of $D$. Then $p_{n}^{n+1} \mathcal{C}$ is a decomposition of $p_{n}^{n+1} D$ and by $\left(B_{n}\right)$ we can take $C \in \mathcal{C}$ such that $p_{n}^{n+1} C$ contains a $V$-box $P$. Let $C_{1}, \ldots, C_{k}$ be the cells in $\mathcal{C}$ such that $p_{n}^{n+1} C=p_{n}^{n+1} C_{i}$ for $i=1, \ldots, k$. After restricting the functions $p_{n}^{n+1} C \rightarrow R$ used to define $C_{1}, \ldots, C_{k}$ to $P$ we see that it is enough to prove the following:

Let $f_{1}, \ldots, f_{m}: P \rightarrow V$ be definable and continuous and let $p, q \in V$ be such that $p<q$ and $|q-p|>\mathfrak{m}$. Then there is a $V$-box $B \subseteq P \times(p, q)$ with $B \cap \Gamma f_{j}=\emptyset$ for all $j$.

For $m=1$ this statement follows from $\left(A_{n}\right)$, and for $m>1$ it follows by a straightforward induction on $m$ using again $\left(A_{n}\right)$.

Corollary 2.5 ( $V$-Box Lemma). Let $X \subseteq R^{n}$ be definable and let $D \subseteq \boldsymbol{k}^{n}$ be a box such that $D \subseteq$ st $X$. Then $X$ contains $a V$-box $B$ with st $B \subseteq D$.

Proof. We may assume that $X \subseteq V^{n}$, and that $\operatorname{cl}(D) \subseteq$ st $X$. Pick a $V$-box $D^{\prime} \subseteq R^{n}$ such that st $D^{\prime}=\operatorname{cl}(D)$, and take a decomposition $\mathcal{C}$ of $R^{n}$ which partitions both $D^{\prime}$ and $X$. By Lemma 2.4, we can take $C \in \mathcal{C}$ such that $C \subseteq D^{\prime}$ and $C$ contains a $V$-box $B$. It is clear that $B \cap X \neq \emptyset$, otherwise $D$ would contain a box whose intersection with st $X$ is empty. So $B \subseteq C \subseteq X$.

Corollary 2.6. If $X \subseteq R^{n}$ is definable, then st $X \cap$ st $X^{c}$ has empty interior in $\boldsymbol{k}^{n}$.

By [1, $\boldsymbol{k}_{\text {ind }}$ is weakly o-minimal. MacPherson, Marker and Steinhorn define in [13] a notion of dimension for weakly o-minimal structures: 
Definition 2.7. Let $M$ be a weakly o-minimal structure, and let $X \subseteq M^{n}$ be definable in $M$. If $X \neq \emptyset$, then $\operatorname{dim}_{w}(X)$ is the largest integer $k \in\{0, \ldots, n\}$ for which there is a projection map

$$
p: M^{n} \rightarrow M^{k}, \quad\left(x_{1}, \ldots, x_{n}\right) \mapsto\left(x_{\lambda(1)}, \ldots, x_{\lambda(k)}\right),
$$

where $1 \leq \lambda(1)<\cdots<\lambda(k) \leq n$, such that $\operatorname{int}(p X) \neq \emptyset$. We set $\operatorname{dim}_{w}(\emptyset)=-\infty$.

Note that if $M$ is o-minimal, then the above notion of dimension agrees with the usual dimension for o-minimal structures.

Corollary 2.8. $\operatorname{dim}_{w}($ st $X) \leq \operatorname{dim} X$ for $V$-bounded $X \in \operatorname{Def}^{n}(R)$.

2.2. Good cells. We define good cells in analogy with [14, and we state some results needed in the proof of good cell decomposition. We omit proofs that are as in [14].

Definition 2.9. Given functions $f: X \rightarrow R$ with $X \subseteq R^{n}$, and $g: C \rightarrow \boldsymbol{k}$ with $C \subseteq \boldsymbol{k}^{n}$, we say that $f$ induces $g$ if $f$ is definable (so $X$ is definable), $C^{h} \subseteq X, f \mid C^{h}$ is continuous, $f\left(C^{h}\right) \subseteq V$ and $\Gamma g=\operatorname{st}(\Gamma f) \cap(C \times \boldsymbol{k})$.

Lemma 2.10. Let $C \subseteq \boldsymbol{k}^{n}$ and suppose $g: C \rightarrow \boldsymbol{k}$ is induced by the function $f: X \rightarrow R$ with $X \subseteq R^{n}$. Then $g$ is continuous.

Proof. Assume towards a contradiction that $g$ is not continuous at $c \in C$. Let $r \in \boldsymbol{k}^{>0}$ be such that for every neighborhood $B \subseteq \boldsymbol{k}^{n}$ of $c$ there is $b \in B \cap C$ with $|g(c)-g(b)| \geq r$. Pick $c^{\prime} \in R^{n}$ with st $\left(c^{\prime}\right)=c$ and define

$$
Y:=\left\{x \in X:\left|f\left(c^{\prime}\right)-f(x)\right| \geq r^{\prime} / 2\right\},
$$

where $r^{\prime} \in R^{>0}$ is such that $\operatorname{st}\left(r^{\prime}\right)=r$. Then $d\left(c^{\prime}, Y\right)$ exists in $R$. If $d\left(c^{\prime}, Y\right)$ is infinitesimal then, since $Y$ is closed, there is $y \in Y$ such that $\operatorname{st}(y)=\operatorname{st}\left(c^{\prime}\right)$, a contradiction with $f$ inducing a function. Hence $d\left(c^{\prime}, Y\right)>\mathfrak{m}$, but this yields a neighborhood $B \subseteq \boldsymbol{k}^{n}$ of $c$ such that $g(B \cap C) \subseteq(g(c)-r, g(c)+r)$, a contradiction.

For $C \subseteq \boldsymbol{k}^{n}$ we let $G(C)$ be the set of all $g: C \rightarrow \boldsymbol{k}$ that are induced by some definable $f: X \rightarrow R$ with $X \subseteq R^{n}$.

Lemma 2.11. Let $1 \leq j(1)<\cdots<j(m) \leq n$ and define $\pi: \boldsymbol{k}^{n} \rightarrow \boldsymbol{k}^{m}$ by

$$
\pi\left(x_{1}, \ldots, x_{n}\right)=\left(x_{j(1)}, \ldots, x_{j(m)}\right) .
$$

Let $C \subseteq \boldsymbol{k}^{n}$ and suppose $g \in G(\pi C)$. Then $\left.g \circ \pi\right|_{C} \in G(C)$.

Definition 2.12. Let $i=\left(i_{1}, \ldots, i_{n}\right)$ be a sequence of zeros and ones. Good $i$-cells are subsets of $\boldsymbol{k}^{n}$ obtained by recursion on $n$ as follows:

(i) For $n=0$ and $i$ the empty sequence, the one-point space $\boldsymbol{k}^{0}$ is the only good $i$-cell, and for $n=1$, a good (0)-cell is a singleton $\{a\}$ with $a \in \boldsymbol{k}$; a good (1)-cell is an interval in $\boldsymbol{k}$. 
(ii) Let $n>0$ and assume inductively that good $i$-cells are subsets of $\boldsymbol{k}^{n}$. A good $(i, 0)$-cell is a set $\Gamma h \subseteq \boldsymbol{k}^{n+1}$ where $h \in G(C)$ and $C \subseteq \boldsymbol{k}^{n}$ is a good $i$-cell. A good $(i, 1)$-cell is either a set $C \times \boldsymbol{k}$, or a set $(-\infty, f) \subseteq \boldsymbol{k}^{n+1}$, or a set $(g, h) \subseteq \boldsymbol{k}^{n+1}$, or a set $(f,+\infty) \subseteq \boldsymbol{k}^{n+1}$, where $f, g, h \in G(C), g<h$, and $C$ is a good $i$-cell.

One verifies easily that a good $i$-cell is open in $\boldsymbol{k}^{n}$ iff $i_{1}=\cdots=i_{n}=1$, and that if $i_{1}=\cdots=i_{n}=1$, then every good $i$-cell is homeomorphic to $\boldsymbol{k}^{n}$. A good cell in $\boldsymbol{k}^{n}$ is a good $i$-cell for some sequence $i=\left(i_{1}, \ldots, i_{n}\right)$ of zeros and ones.

Lemma 2.13. Let $C \subseteq \boldsymbol{k}^{n}$ be a good $\left(i_{1}, \ldots, i_{n}\right)$-cell, and let $k \in\{1, \ldots, n\}$ be such that $i_{k}=0$. Let $\pi: \boldsymbol{k}^{n} \rightarrow \boldsymbol{k}^{n-1}$ be given by

$$
\pi\left(x_{1}, \ldots, x_{n}\right)=\left(x_{1}, \ldots, x_{k-1}, x_{k+1}, \ldots, x_{n}\right) .
$$

Then $\pi(C) \subseteq \boldsymbol{k}^{n-1}$ is a good cell, $\pi \mid C: C \rightarrow \pi(C)$ is a homeomorphism, and if $E \subseteq \pi(C)$ is a good cell, so is its inverse image $\pi^{-1}(E) \cap C$.

2.3. More on good cells. We prove here that $(R, V) \models \mathcal{I}$ iff $(R, V) \models$ $\Sigma_{\mathrm{i}}$ (see page 116 for definitions of $\mathcal{I}$ and $\Sigma_{\mathrm{i}}$ ). This implies that if $(R, V) \models \Sigma_{\mathrm{i}}$, then good cells in $\boldsymbol{k}^{n}$ are differences of standard parts of definable subsets of $R^{n}$.

It is not difficult to show that if $(R, V) \models \mathcal{I}$, then for all $n$ and all definable $X, Y \subseteq R^{n}$ there is a definable $Z \subseteq R^{n}$ such that st $X \cap$ st $Y=$ st $Z$ : Set $J(\boldsymbol{k}):=(-1,1) \subseteq \boldsymbol{k}$ and $J:=(-1,1) \subseteq R$. We shall use the definable homeomorphism

$$
\tau_{n}: R^{n} \rightarrow J^{n}:\left(x_{1} \ldots, x_{n}\right) \mapsto\left(\frac{x_{1}}{\sqrt{1+x_{1}^{2}}}, \ldots, \frac{x_{n}}{\sqrt{1+x_{n}^{2}}}\right),
$$

and we also let $\tau_{n}$ denote the homeomorphism

$$
\tau_{n}: \boldsymbol{k}^{n} \rightarrow J(\boldsymbol{k})^{n}:\left(x_{1} \ldots, x_{n}\right) \mapsto\left(\frac{x_{1}}{\sqrt{1+x_{1}^{2}}}, \ldots, \frac{x_{n}}{\sqrt{1+x_{n}^{2}}}\right) .
$$

One easily checks that $\tau_{1}: R \rightarrow J$ induces $\tau_{1}: \boldsymbol{k} \rightarrow J(\boldsymbol{k})$, and that for $X \in \operatorname{Def}^{n}(R)$,

$$
\tau_{n}(\operatorname{st} X)=\operatorname{st}\left(\tau_{n} X\right) \cap J(\boldsymbol{k})^{n} ;
$$

moreover,

$$
\tau_{n}^{-1}\left(\operatorname{st}(X) \cap J(\boldsymbol{k})^{n}\right)=\operatorname{st}\left(\tau_{n}^{-1}(X)\right) \quad \text { for } X \subseteq J(\boldsymbol{k})^{n},
$$

where $\tau_{n}^{-1}: J^{n} \rightarrow R^{n}$ and $\tau_{n}^{-1}: J(\boldsymbol{k})^{n} \rightarrow \boldsymbol{k}^{n}$ are the inverse functions of $\tau_{n}: R^{n} \rightarrow J^{n}$ and of $\tau_{n}: \boldsymbol{k}^{n} \rightarrow J(\boldsymbol{k})^{n}$ respectively.

Suppose $(R, V)$ satisfies $\mathcal{I}$. To see that then for all $n$ and all $X, Y \in$ $\operatorname{Def}^{n}(R)$ there is $Z \in \operatorname{Def}^{n}(R)$ such that st $X \cap$ st $Y=$ st $Z$, let $X, Y \in$ 
$\operatorname{Def}^{n}(R)$. Then $\tau_{n} X, \tau_{n} Y \subseteq J^{n}$, so we can take $Z \in \operatorname{Def}^{n}(R)$ such that

$$
\operatorname{st}\left(\tau_{n} X\right) \cap \operatorname{st}\left(\tau_{n} Y\right)=\operatorname{st} Z \text {. }
$$

We claim that

$$
\text { st } X \cap \operatorname{st} Y=\operatorname{st}\left(\tau_{n}^{-1}\left(Z \cap J^{n}\right)\right) \text {. }
$$

To prove this it is enough to show that

$$
\tau_{n}(\text { st } X \cap \operatorname{st} Y)=\tau\left(\operatorname{st}\left(\tau_{n}^{-1}\left(Z \cap J^{n}\right)\right)\right) .
$$

Now the right-hand side of (1) is equal to

$$
\operatorname{st}\left(Z \cap J^{n}\right) \cap J(\boldsymbol{k})^{n}=\operatorname{st}(Z) \cap J(\boldsymbol{k})^{n},
$$

and we have

$$
\tau_{n}(\text { st } X \cap \operatorname{st} Y)=\operatorname{st}\left(\tau_{n} X\right) \cap \operatorname{st}\left(\tau_{n} Y\right) \cap J(\boldsymbol{k})^{n} .
$$

In view of $\operatorname{st}\left(\tau_{n} X\right) \cap \operatorname{st}\left(\tau_{n} Y\right)=\operatorname{st} Z$ this gives (1).

In a similar way the condition $\Sigma_{\mathrm{i}}$ implies its "unrestricted version", i.e. the variant obtained by substituting $R$ for $I$. We shall often use these facts tacitly.

Lemma 2.14. Suppose $(R, V)$ satisfies $\mathcal{I}$. Then $(R, V) \models \Sigma_{\mathrm{i}}$.

Proof. Let $X \subseteq I^{1+n}$ be definable and increasing in the first variable. Towards proving that $X$ satisfies the conclusion of $\Sigma_{\mathrm{i}}$ we may assume that $X$ is closed.

Claim 1. There is $\epsilon_{0} \in \mathfrak{m} \geq 0$ such that

$$
\operatorname{st}(X) \cap\left(\{0\} \times I(\boldsymbol{k})^{n}\right)=\operatorname{st}\left(X \cap\left(\left[0, \epsilon_{0}\right] \times I^{n}\right)\right) .
$$

We set $Y:=\{0\} \times I^{n}$ and take a definable $Z \subseteq I^{n+1}$ with st $X \cap$ st $Y=$ st $Z$. We may assume that $Z$ is closed and nonempty, and we set $\epsilon_{1}:=$ $\sup \{d(z, X): z \in Z\}$ and $\epsilon_{2}:=\sup \{d(z, Y): z \in Z\}$. Then $\epsilon_{1}, \epsilon_{2} \in \mathfrak{m} \geq 0$, and we claim that $\epsilon_{0}:=\epsilon_{1}+\epsilon_{2}$ works. Clearly,

$$
\operatorname{st}\left(X \cap\left(\left[0, \epsilon_{0}\right] \times I^{n}\right)\right) \subseteq \operatorname{st}(X) \cap\left(\{0\} \times I(\boldsymbol{k})^{n}\right) .
$$

So let $a \in \operatorname{st} X \cap \operatorname{st} Y$. Then $a=\operatorname{st}(z)$ with $z \in Z$. We have $d(z, X) \leq \epsilon_{1}$ and $d(z, Y) \leq \epsilon_{2}$. Since $Z$ is closed and $V$-bounded, we can take $x \in X$ and $y \in Y$ such that $d(x, z) \leq \epsilon_{1}, d(y, z) \leq \epsilon_{2}$. Then $d(x, y) \leq \epsilon_{1}+\epsilon_{2}=\epsilon_{0}$, and it follows that

$$
a=\operatorname{st}(x) \in \operatorname{st}\left(X \cap\left(\left[0, \epsilon_{0}\right] \times I^{n}\right)\right) .
$$

This proves Claim 1. Let $\epsilon_{0}$ be as in Claim 1 .

Claim 2. st $X(\epsilon)=\operatorname{st} X\left(\epsilon_{0}\right)$ for all $\epsilon \in \mathfrak{m} \geq \epsilon_{0}$.

It is clear that st $X\left(\epsilon_{0}\right) \subseteq$ st $X(\epsilon)$ for all $\epsilon \in \mathfrak{m}^{\geq \epsilon_{0}}$. To prove the other inclusion, let $a \in \operatorname{st} X(\epsilon)$. Then

$$
(0, a) \in \operatorname{st}(X) \cap\left(\{0\} \times I(\boldsymbol{k})^{n}\right),
$$


hence

$$
(0, a) \in \operatorname{st}\left(X \cap\left(\left[0, \epsilon_{0}\right] \times I^{n}\right)\right)
$$

by Claim 1 . Because $X$ is increasing in the first variable, this implies $(0, a) \in$ st $X\left(\epsilon_{0}\right)$.

LemMa 2.15. $\Sigma_{\mathrm{i}} \Rightarrow \mathcal{I}$.

Proof. Suppose $(R, V)$ satisfies $\Sigma_{\mathrm{i}}$. Let $X, Y \subseteq I^{n}$ be definable and nonempty. For $\epsilon \in R^{\geq 0}$ define

$$
Y^{\epsilon}:=\left\{x \in R^{n}: d(x, Y) \leq \epsilon\right\} .
$$

We claim that

$$
\bigcup_{\epsilon} \operatorname{st}\left(X \cap Y^{\epsilon}\right)=\operatorname{st} X \cap \operatorname{st} Y,
$$

where $\epsilon$ ranges over all positive infinitesimals. If $a \in \operatorname{st}\left(X \cap Y^{\epsilon}\right)$, then clearly $a \in \operatorname{st} X$ and $a \in$ st $Y$. If $a \in \operatorname{st} X \cap$ st $Y$, then we can take $a^{\prime} \in X$ and $a^{\prime \prime} \in Y$ such that $\operatorname{st}\left(a^{\prime}\right)=\operatorname{st}\left(a^{\prime \prime}\right)=a$ and $d\left(a^{\prime}, a^{\prime \prime}\right)<\epsilon$ for some $\epsilon \in \mathfrak{m}^{>0}$. Hence $a^{\prime} \in X \cap Y^{\epsilon}$.

Now by $\Sigma_{\mathrm{i}}$, there is a positive infinitesimal $\epsilon_{0}$ such that

$$
\operatorname{st}\left(X \cap Y^{\epsilon_{0}}\right)=\bigcup_{\epsilon} \operatorname{st}\left(X \cap Y^{\epsilon}\right) .
$$

The proofs of the following two lemmas are similar to the proofs of their counterparts in [14].

Lemma 2.16. Suppose $(R, V)$ satisfies $\mathcal{I}$, and let $X \subseteq R^{n}$ and $f: X \rightarrow R$ be definable, and put

$$
X^{-}:=\{x \in X: f(x)<V\}, \quad X^{+}:=\{x \in X: f(x)>V\} .
$$

Then st $X^{-}$and st $X^{+}$belong to $\mathrm{St}_{n}$.

Corollary 2.17. If $(R, V)$ satisfies $\mathcal{I}$, and $X \subseteq R^{n}$ and $g: X \rightarrow R$ are definable, then $\operatorname{st}\{x \in X: g(x) \in \mathfrak{m}\} \in \mathrm{St}_{n}$.

Conversely, if the conclusion of this corollary holds for all $n$ and definable $g: X \rightarrow R$ with $X \subseteq R^{n}$, then $(R, V)$ satisfies $\mathcal{I}$. To see this, let $X, Y \subseteq V^{n}$ be definable with $Y \neq \emptyset$. Assume the conclusion of the corollary holds for the function $x \mapsto d(x, Y): X \rightarrow R$. Then we have a definable $Z \subseteq V^{n}$ such that st $Z=\operatorname{st}\{x \in X: d(x, Y) \in \mathfrak{m}\}$. This gives st $X \cap$ st $Y=$ st $Z$.

From now on until the end of Section 2 we assume $(R, V) \models \Sigma_{\mathrm{i}}$. The following lemma is now proved as in [14].

Lemma 2.18. Every good cell in $\boldsymbol{k}^{n}$ is of the form $X \backslash Y$ with $X, Y \in \mathrm{St}_{n}$. 
2.4. Good cell decomposition. We obtain good cell decomposition, namely, if $X_{1}, \ldots, X_{m} \subseteq R^{n}$ are definable, then there is a finite partition of $\boldsymbol{k}^{n}$ into good cells that partitions every st $X_{i}$. A consequence of this is that the $\boldsymbol{k}_{\text {ind }}$-definable subsets of $\boldsymbol{k}^{n}$ are finite unions of differences st $X \backslash$ st $Y$, where $X, Y \in \operatorname{Def}^{n}(R)$.

The proof of the following lemma is again as in [14].

Lemma 2.19. Let $C \subseteq \boldsymbol{k}^{n}$ be a good $i$-cell, let $X \subseteq R^{n+1}$ be definable and suppose $k \in\{1, \ldots, n\}$ is such that $i_{k}=0$. Define $\pi: \boldsymbol{k}^{n+1} \rightarrow \boldsymbol{k}^{n}$ by

$$
\pi(x)=\left(x_{1}, \ldots, x_{k-1}, x_{k+1}, \ldots, x_{n+1}\right) .
$$

Then $\pi(\operatorname{st}(X) \cap(C \times \boldsymbol{k}))$ is a difference of sets in $\mathrm{St}_{n}$.

A good decomposition of $I(\boldsymbol{k})^{n}$ is a special kind of partition of $I(\boldsymbol{k})^{n}$ into finitely many good cells. The definition is by recursion on $n$ :

(i) a good decomposition of $I(\boldsymbol{k})$ is a collection

$$
\left\{\left(c_{0}, c_{1}\right),\left(c_{1}, c_{2}\right), \ldots,\left(c_{k}, c_{k+1}\right),\left\{c_{0}\right\},\left\{c_{1}\right\}, \ldots,\left\{c_{k}\right\},\left\{c_{k+1}\right\}\right\}
$$

of intervals and points in $\boldsymbol{k}$ where $c_{0}<c_{1}<\cdots<c_{k}<c_{k+1}$ are real numbers with $c_{0}=-1$ and $c_{k+1}=1$;

(ii) a good decomposition of $I(\boldsymbol{k})^{n+1}$ is a finite partition $\mathcal{D}$ of $I(\boldsymbol{k})^{n+1}$ into good cells such that $\left\{\pi_{n}^{n+1} C: C \in \mathcal{D}\right\}$ is a good decomposition of $I(\boldsymbol{k})^{n}$.

Theorem 2.20 (Good Cell Decomposition).

$\left(A_{n}\right)$ Given any definable $X_{1}, \ldots, X_{m} \subseteq I^{n}$, there is a good decomposition of $I(\boldsymbol{k})^{n}$ partitioning each set st $X_{i}$.

$\left(B_{n}\right)$ If $f: X \rightarrow I$, with $X \subseteq I^{n}$, is definable, then there is a good decomposition $\mathcal{D}$ of $I(\boldsymbol{k})^{n}$ such that for every open $C \in \mathcal{D}$, either the set st $(\Gamma f) \cap(C \times \boldsymbol{k})$ is empty, or $f$ induces a function $g: C \rightarrow I(\boldsymbol{k})$.

The proof uses the lemmas above and is very similar to that of Theorem 4.3 in [14.

A good decomposition of $\boldsymbol{k}^{n}$ is a special kind of partition of $\boldsymbol{k}^{n}$ into finitely many good cells. The definition is by recursion on $n$ :

(i) a good decomposition of $\boldsymbol{k}^{1}=\boldsymbol{k}$ is a collection

$$
\left\{\left(c_{0}, c_{1}\right),\left(c_{1}, c_{2}\right), \ldots,\left(c_{k}, c_{k+1}\right),\left\{c_{1}\right\}, \ldots,\left\{c_{k}\right\}\right\}
$$

of intervals and points in $\boldsymbol{k}$, where $c_{1}<\cdots<c_{k} \in \boldsymbol{k}$ and $c_{0}=-\infty$, $c_{k+1}=\infty$

(ii) a good decomposition of $\boldsymbol{k}^{n+1}$ is a finite partition $\mathcal{D}$ of $\boldsymbol{k}^{n+1}$ into good cells such that $\left\{\pi_{n}^{n+1} C: C \in \mathcal{D}\right\}$ is a good decomposition of $\boldsymbol{k}^{n}$.

The following corollary and theorem are proved just as in [14]. 
COROllary 2.21. If $X_{1}, \ldots, X_{m} \subseteq R^{n}$ are definable, then there is a good decomposition of $\boldsymbol{k}^{n}$ partitioning every st $X_{i}$.

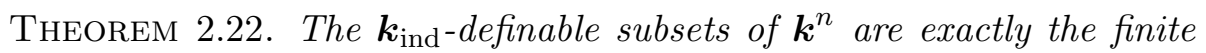
unions of sets st $X \backslash$ st $Y$ with $X, Y \in \operatorname{Def}^{n}(R)$.

As in [14] we find that the standard part of a partial derivative of a definable function is almost everywhere equal to the corresponding partial derivative of the standard part of the function:

Theorem 2.23. Let $f: Y \rightarrow R$ with $Y \subseteq R^{n}$ be definable with $V$-bounded graph. Then there is a good decomposition $\mathcal{D}$ of $\boldsymbol{k}^{n}$ that partitions st $Y$ such that if $D \in \mathcal{D}$ is open and $D \subseteq$ st $Y$, then $f$ is continuously differentiable on an open definable $X \subseteq Y$ containing $D^{h}$, and $f, \partial f / \partial x_{1}, \ldots, \partial f / \partial x_{n}$, as functions on $X$, induce functions $g, g_{1}, \ldots, g_{n}: D \rightarrow \boldsymbol{k}$ such that $g$ is $C^{1}$ and $g_{i}=\partial g / \partial x_{i}$ for all $i$.

3. The conditions $\mathcal{C}, \Sigma_{\mathrm{i}}, \Sigma_{\mathrm{d}}$ and $\Sigma$. In this section we show that $\Sigma_{\mathrm{i}} \& \Sigma_{\mathrm{d}}$ implies $\mathcal{C}$, we prove that various conditions imply $\Sigma$, and we give an example showing that $\boldsymbol{k}_{\text {ind }}$ is not always o-minimal.

3.1. Closed and definably connected sets. The conditions $\Sigma_{\mathrm{d}}$ and $\mathcal{C}$ on pairs $(R, V)$ are stated on page 116 . Note that if $(R, V)$ satisfies $\mathcal{C}$, then $\boldsymbol{k}_{\text {ind }}$ is o-minimal by Lemma 2.3 . For $(R, V)$ to satisfy $\mathcal{C}$ it suffices that for each $n$ the closed $\boldsymbol{k}_{\text {ind }}$-definable subsets of $I(\boldsymbol{k})^{n}$ are exactly the sets st $X$ with definable $X \subseteq I^{n}$. (This follows by means of the homeomorphisms $\tau_{n}$.)

Proposition 3.1. Suppose $(R, V) \models \Sigma_{\mathrm{i}}$ and $(R, V) \models \Sigma_{\mathrm{d}}$. Then $(R, V)$ satisfies $\mathcal{C}$. (In particular, $\Sigma \Rightarrow \mathcal{C}$.)

Proof. The result will follow from Corollary 2.21 once we show that the closure of a good cell in $\boldsymbol{k}^{n}$ is of the form st $X$ for some definable $X \subseteq R^{n}$. Let $\epsilon$ range over all positive infinitesimals, and let $C \subseteq \boldsymbol{k}^{n}$ be a good cell.

Claim. There is $r_{0} \in R^{>\mathfrak{m}}$ and a definable $X \subseteq\left(0, r_{0}\right) \times R^{n}$ such that

$$
0<r<r^{\prime}<r_{0} \Rightarrow X\left(r^{\prime}\right) \subseteq X(r), \quad \operatorname{st}\left(\bigcap_{\epsilon} X(\epsilon)\right)=C,
$$

where $\epsilon$ ranges over all positive infinitesimals.

This claim follows by the same argument as the corresponding claim in the proof of Proposition 5.1 in [14. Let $X \subseteq\left(0, r_{0}\right) \times R^{n}$ be as in the Claim. Then, since $(R, V) \models \Sigma_{\mathrm{d}}$, we can take $\epsilon \in \mathfrak{m}^{>0}$ such that st $X(\epsilon)=\operatorname{cl}(C)$.

For $Z \subseteq V^{n}$ we let $Z^{h}:=\mathrm{st}^{-1}$ (st $\left.Z\right)$.

Proposition 3.2. Suppose $(R, V)$ satisfies $\mathcal{C}$, and let $X \subseteq V^{n}$ be definable and definably connected in $R$. Then st $X$ is definably connected. 
Proof. Assume to the contrary that st $X$ is not definably connected. Then st $X=$ st $Y_{1} \dot{\cup}$ st $Y_{2}$ for some definable, nonempty $Y_{1}, Y_{2} \subseteq R^{n}$. We may assume that $Y_{1}, Y_{2}$ are closed. Let

$$
q:=\inf \left\{d\left(y, \text { st } Y_{2}\right): y \in \operatorname{st} Y_{1}\right\} .
$$

Since st $Y_{1}$, st $Y_{2}$ are closed and bounded, $q \in \boldsymbol{k}^{>0}$. Define

$$
X_{1}:=\left\{x \in R^{n}: d\left(x, Y_{1}\right) \leq q / 4\right\} \quad \text { and } \quad X_{2}:=\left\{x \in R^{n}: d\left(x, Y_{2}\right) \leq q / 4\right\} .
$$

Then $X_{1}, X_{2}$ are closed and disjoint, and $Y_{1}^{h} \subseteq X_{1}, Y_{2}^{h} \subseteq X_{2}$. Since $X^{h}=$ $Y_{1}^{h} \cup Y_{2}^{h}$, we have $X=\left(X \cap X_{1}\right) \cup\left(X \cap X_{2}\right)$, where $X \cap X_{1}, X \cap X_{2}$ are nonempty, disjoint, and closed in $X$, a contradiction with $X$ being definably connected.

3.2. Conditions implying $\Sigma$. In the next lemma we use the following convention. Let $C \subseteq R^{n}$ be an $\left(i_{1}, \ldots, i_{n}\right)$-cell of dimension $k$. Let $\lambda:\{1, \ldots, n\} \rightarrow\{1, \ldots, n\}$ be such that

$$
1 \leq \lambda(1)<\cdots<\lambda(k) \leq n
$$

and $i_{\lambda(1)}=\cdots=i_{\lambda(k)}=1$. We define

$$
C_{0}:=\left\{a \in R^{k} \text { : there is } x \in C \text { such that } x_{\lambda(1)}=a_{1} \& \ldots \& x_{\lambda(k)}=a_{k}\right\} .
$$

Then $C_{0}$ is the homeomorphic image of $C$ under a coordinate projection $p: R^{n} \rightarrow R^{k}$. For a definable $C^{1}$-function $f: C \rightarrow R$ we let $\hat{f}: C_{0} \rightarrow R$ be defined by $\hat{f}(p(x))=f(x)$ where $x \in C$. We denote by $\frac{\partial f}{\partial x_{j}}(a)$, where $a \in C$ and $j \in\{1, \ldots, k\}$, the $j$ th partial derivative of $\hat{f}$ at $p(a)$.

Lemma 3.3. Suppose cofinality $(\mathfrak{m})>2^{|\boldsymbol{k}|}$. Then $(R, V)$ satisfies $\Sigma$.

Proof. Let $X \in \operatorname{Def}^{1+n}(R)$. By cell decomposition we may assume that $X$ is an $\left(i_{1}, \ldots, i_{n+1}\right)$-cell satisfying for every $k=1, \ldots, n+1$ the following: If $p_{k}^{n+1} X=(f, g)$, then all $\partial f / \partial x_{i}, \partial g / \partial x_{i}$ have constant sign on $p_{k-1}^{n+1} X$. If $p_{k}^{n+1} X=\Gamma f$, then all $\partial f / \partial x_{i}$ have constant sign on $\left(p_{k-1}^{n+1} X\right)_{0}$.

Now there are $2^{|\boldsymbol{k}|}$ distinct subsets of $\boldsymbol{k}^{n}$. Let $f: \mathfrak{m}^{>0} \rightarrow \mathcal{P}\left(\boldsymbol{k}^{n}\right)$, where $\mathcal{P}\left(\boldsymbol{k}^{n}\right)$ is the power set of $\boldsymbol{k}^{n}$, be given by $\epsilon \mapsto$ st $X(\epsilon)$. Assume to the contrary that for every $\epsilon_{1} \in \mathfrak{m}^{>0}$ we can find $\epsilon_{2} \in \mathfrak{m}^{>\epsilon_{1}}$ such that st $X\left(\epsilon_{1}\right) \neq$ st $X\left(\epsilon_{2}\right)$. Then the above assumption on $X$ yields a cofinal subset of $\mathfrak{m}$ such that $f$ is injective on this subset, a contradiction.

Note that, together with 5.3 and 6.4 in [5], this lemma implies that if $V$ is a $T$-convex subring of $R$, then $(R, V) \models \Sigma$.

Lemma 3.4. Let $R$ be $\omega$-saturated. Then $(R, \mathcal{O}) \models \Sigma$.

Proof. Let $X \subseteq R^{1+n}$ be defined over $a \in R^{k}$. Since $R$ is $\omega$-saturated, we can take $\epsilon \in \mathfrak{m}$ such that $\epsilon>\delta$ for every $\delta \in \operatorname{dcl}(a)$ with $\delta<\mathbb{Q}^{>0}$. Then for every $\epsilon^{\prime} \in \mathfrak{m}^{>\epsilon}, \operatorname{tp}\left(\epsilon^{\prime} \mid a\right)=\operatorname{tp}(\epsilon \mid a)$, and, in particular, st $X\left(\epsilon^{\prime}\right)=\operatorname{st} X(\epsilon)$. 
Otherwise we could find $x \in \operatorname{st} X\left(\epsilon^{\prime}\right) \triangle$ st $X(\epsilon)$ and a box $B=\left(p_{1}, q_{1}\right) \times \cdots \times$ $\left(p_{n}, q_{n}\right) \subseteq \mathbb{R}^{n}$ with $p_{i}, q_{i} \in \mathbb{Q}$ such that $x \in B$ and either $\operatorname{cl}(B) \cap$ st $X(\epsilon)=\emptyset$ or $\operatorname{cl}(B) \cap$ st $X\left(\epsilon^{\prime}\right)=\emptyset$. Then $B^{\prime}=\left(p_{1}, q_{1}\right) \times \cdots \times\left(p_{n}, q_{n}\right) \subseteq R^{n}$ is such that $B^{\prime} \cap X(\epsilon)=\emptyset$ and $B^{\prime} \cap X\left(\epsilon^{\prime}\right) \neq \emptyset$, or vice versa, a contradiction.

We saw in Section 2 that if $(R, V) \models \Sigma_{\mathrm{i}}$, then $\boldsymbol{k}_{\text {ind }}$ is o-minimal. However, the following example shows that $\boldsymbol{k}_{\text {ind }}$ is not always o-minimal.

ExAmple. Let $\mathbb{R}_{\exp }$ be the real exponential field and let $R$ be a proper elementary extension. Take $\lambda \in R$ such that $\lambda>\mathbb{R}$, and let $V$ be the smallest convex subring of $R$ containing $\lambda$, i.e.

$$
V:=\left\{y:|y|<\lambda^{n} \text { for some } n\right\},
$$

and let $\boldsymbol{k}$ be the corresponding residue field. We define $\log : R^{>0} \rightarrow R$ to be the inverse function of exp: $R \rightarrow R^{>0}$. Then $\log \left(V^{>0}\right)=V$ and it induces an increasing and injective function $\boldsymbol{k}^{>0} \rightarrow \boldsymbol{k}$, which, for simplicity, we shall also denote by $\log$. Now the set $\left\{\operatorname{st}(\lambda)^{n}: n \in \mathbb{N}\right\}$ is cofinal in $\boldsymbol{k}^{>0}$, hence $\left\{\log \operatorname{st}(\lambda)^{n}: n \in \mathbb{N}\right\}$ is cofinal in $\log \boldsymbol{k}^{>0}$. So the set $\log \boldsymbol{k}^{>0}$ is definable in $\boldsymbol{k}_{\text {ind }}$, but, because $\log \operatorname{st}(\lambda)^{n}=n \log \operatorname{st}(\lambda)$, it is not cofinal in $\boldsymbol{k}^{>0}$, nor does it have a supremum. It follows that $\boldsymbol{k}_{\text {ind }}$ cannot be o-minimal, nor does $(R, V)$ satisfy $\Sigma_{\mathrm{i}}$.

4. Traces. Recall from the Introduction that a set $X \subseteq R^{n}$ is a trace if $X=Y \cap R^{n}$ for some $n$-ary relation $Y$ defined in some elementary extension of $R$ using parameters from that extension. Note that every $X \in \operatorname{Def}^{n}(R)$ is a trace, and that if $X, Y \subseteq R^{n}$ are traces, then so are $X \cup Y, X \cap Y$ and $X^{c}$. An example of a trace is $V \subseteq R$ : take an element $\lambda$ in an elementary extension of $R$ such that $V<\lambda<R^{>V}$. Then $V=(-\lambda, \lambda) \cap R$ where the interval $(-\lambda, \lambda)$ is taken in the extension.

We let $R^{*}$ be the expansion of $R$ by all traces $X \subseteq R^{n}$, for all $n$. By the main result of [1] every subset of $R^{n}$ definable in $R^{*}$ is a trace. It follows that every subset of $R^{n}$ definable in $(R, V)$ is a trace.

LEMma 4.1. Let $\boldsymbol{k}^{*}$ be the expansion of the ordered field $\boldsymbol{k}$ by the sets st $X \subseteq \boldsymbol{k}^{n}$ for all traces $X \subseteq R^{n}$ and all $n$. Then, for all $n$,

$$
\operatorname{Def}^{n}\left(\boldsymbol{k}^{*}\right)=\left\{\text { st } X: X \subseteq R^{n} \text { is a trace }\right\} .
$$

Proof. We first show that for every $n$, the collection

$$
\mathcal{C}_{n}:=\left\{\text { st } X: X \subseteq R^{n} \text { is a trace }\right\}
$$

is a boolean algebra on $\boldsymbol{k}^{n}$. It is clear that

$$
\text { st } X_{1} \cup \text { st } X_{2}=\operatorname{st}\left(X_{1} \cup X_{2}\right)
$$

for all traces $X_{1}, X_{2} \subseteq R^{n}$. To see that $\mathcal{C}_{n}$ is closed under complements, let 
$X \subseteq R^{n}$ be a trace, and note that

$$
(\operatorname{st} X)^{c}=\operatorname{st}\left\{y \in R^{n}: d(y, x)>\mathfrak{m} \text { for every } x \in X\right\} .
$$

Since $\mathfrak{m}$ is a trace, the set $\left\{y \in R^{n}: d(y, x)>\mathfrak{m}\right.$ for all $\left.x \in X\right\}$ is definable in $R^{*}$, hence, by [1], it is itself a trace. We conclude that the sets st $X$, where $X \subseteq R^{n}$ is a trace, are the elements of a boolean algebra on $\boldsymbol{k}^{n}$.

Now let $X \subseteq R^{n}$ be a trace, and let $0 \leq m \leq n$. We may assume that $X \subseteq V^{n}$ (since $V$ is a trace). Then $\pi_{m}^{n}($ st $X)=\operatorname{st}\left(p_{m}^{n} X\right)$, and by [1, $p_{m}^{n} X$ is a trace.

It follows from Lemma 4.1 and [2] that $\boldsymbol{k}^{*}$ is weakly o-minimal.

LEMMA 4.2. Let $S_{1}$ be a weakly o-minimal structure and $S_{2}$ an o-minimal structure on the same underlying ordered set $S$. Suppose for every $n$ and for every $X_{1} \in \operatorname{Def}^{n}\left(S_{1}\right)$ there is $X_{2} \in \operatorname{Def}^{n}\left(S_{2}\right)$ such that $X_{1} \triangle X_{2}$ has empty interior in $S^{n}$. Then $\operatorname{Def}^{n}\left(S_{1}\right) \subseteq \operatorname{Def}^{n}\left(S_{2}\right)$ for all $n$.

Proof. We proceed by induction on $n$. Let $n=1$. If $X \subseteq S$ is a finite union of convex sets, and $Y \subseteq S$ is a finite union of points and intervals, then either $X \triangle Y$ is finite, or $X \triangle Y$ has nonempty interior. It follows that $\operatorname{Def}^{1}\left(S_{1}\right) \subseteq \operatorname{Def}^{1}\left(S_{2}\right)$ and, in particular, $S_{1}$ is o-minimal.

So assume $\operatorname{Def}^{k}\left(S_{1}\right) \subseteq \operatorname{Def}^{k}\left(S_{2}\right)$ for $k=1, \ldots, n$. Since $S_{1}$ and $S_{2}$ are o-minimal, it suffices to show that every $S_{1}$-cell in $S^{n+1}$ is definable in $S_{2}$. It is even enough to prove this for $S_{1}$-cells $\Gamma g$; here $g: C \rightarrow S$ is a continuous and $S_{1}$-definable function on an $S_{1}$-cell $C \subseteq S^{n}$. Let $\Gamma g$ be such an $S_{1}$-cell.

First, suppose $C$ is an open cell. By the inductive assumption $C \in$ $\operatorname{Def}^{n}\left(S_{2}\right)$ and we can take $X \in \operatorname{Def}^{n+1}\left(S_{2}\right)$ with $X \subseteq C \times S$ such that $(-\infty, g) \triangle X$ does not contain a box. Let $p: S^{n+1} \rightarrow S^{n}$ be given by $p\left(x_{1}, \ldots, x_{n+1}\right)=\left(x_{1}, \ldots, x_{n}\right)$. For $X, Y \subseteq S^{n+1}$ we say that $X<Y$ if for all $a \in S^{n}$ and $(a, x) \in X,(a, y) \in Y$ we have $x<y$. Now take an $S_{2}$-decomposition $\mathcal{D}$ of $S^{n+1}$ which partitions $X$, and let $C_{1}, \ldots, C_{k}$ be the open cells in $p \mathcal{D}$ with $C_{i} \subseteq p X$. We claim that $\Gamma\left(g \mid C_{i}\right) \in \operatorname{Def}^{n+1}\left(S_{2}\right)$ for every $i$.

So let $i \in\{1, \ldots, k\}$, and let $D_{1}, \ldots, D_{l}$ be the open cells in $\mathcal{D}$ with $D_{j} \subseteq X$ and $p D_{j}=C_{i}$ for all $j$. If $D_{j}=\left(f_{j}, g_{j}\right)$ and $D_{j} \cap \Gamma\left(g \mid C_{i}\right) \neq \emptyset$ for some $j \in\{1, \ldots, l\}$, then there is $x \in C_{i}$ with $g(x)<g_{j}(x)$. Then, by continuity of $g$ and $g_{j}$, we obtain a box $B \subseteq X \backslash(-\infty, g)$, a contradiction. So $D_{j} \cap \Gamma g=\emptyset$, and, in particular, $D_{j}<\Gamma\left(g \mid C_{i}\right)$ for every $j$.

Let $d \in\{1, \ldots, l\}$ be such that $D_{j}<D_{d}=\left(f_{d}, g_{d}\right)$ for all $j \neq d$. If $g_{d}<$ $g \mid C_{i}$ on a subset of $C_{i}$ with nonempty interior, then, again by continuity of $g$ and $g_{d}$, we find a box $B \subseteq(-\infty, g)$ with $\Gamma\left(g_{d} \mid p B\right)<B$. Since $B$ intersects $X$ in only at most finitely many cells of the form $\Gamma h$, where $h: C_{i} \rightarrow S$ is continuous, we can find a box $B^{\prime} \subseteq(-\infty, g) \backslash X$, a contradiction. So 
$g_{d}=g \mid C_{i}$ outside a subset of $C_{i}$ with empty interior, hence $g_{d}=g \mid C_{i}$ by continuity of $g$ and $g_{d}$.

We have shown that $\Gamma\left(g \mid C_{i}\right)$ is $S_{2}$-definable for all $i=1, \ldots, k$. It is easy to check that then

$$
\Gamma g=\operatorname{cl}\left(\bigcup_{i=1}^{k} \Gamma\left(g \mid C_{i}\right)\right) \cap\left(C_{i} \times S\right),
$$

hence $\Gamma g \in \operatorname{Def}^{n+1}\left(S_{2}\right)$.

So let $\Gamma g \in \operatorname{Def}^{n+1}\left(S_{2}\right)$ be an $\left(i_{1}, \ldots, i_{n}, 0\right)$-cell with $i_{k}=0$ where $1 \leq$ $k \leq n$, and let

$$
q: S^{n+1} \rightarrow S^{n}:\left(x_{1}, \ldots, x_{n+1}\right) \mapsto\left(x_{1}, \ldots, x_{k-1}, x_{k+1}, \ldots, x_{n+1}\right) .
$$

By the inductive assumption, $q(\Gamma g) \in \operatorname{Def}^{n}\left(S_{2}\right)$. We define $\Gamma g$ in $S_{2}$ as

$$
\left\{(x, y): x \in C \text { and }\left(x_{1}, \ldots, x_{k-1}, x_{k+1}, \ldots, x_{n}, y\right) \in q(\Gamma g)\right\} .
$$

The main result of this section is Theorem 4.4, where we assume that $R$ is $\omega$-saturated and $V=\mathcal{O}$. This assumption is essential: Suppose $\boldsymbol{k}_{\text {ind }}$ is o-minimal but $\boldsymbol{k}$ is not isomorphic to $\mathbb{R}$. Then $\boldsymbol{k}$ has a nonempty bounded convex subset $X$ without a least upper bound in $\boldsymbol{k}$, so $X$ is not definable in $\boldsymbol{k}_{\text {ind }}$. However, $X^{h} \subseteq R$ is a trace, and so $X=$ st $Y$ for some trace set $Y \subseteq R^{n}$.

In the rest of this section we assume that $R$ is $\omega$-saturated and $V=\mathcal{O}$. In particular, $\boldsymbol{k}=\mathbb{R}$.

Lemma 4.3. Let $Y \subseteq R^{n}$ be a trace. Then there is a definable $Z \subseteq R^{n}$ such that st $Y \triangle$ st $Z$ has empty interior in $\mathbb{R}^{n}$.

Proof. Take an elementary extension $R^{\prime}$ of $R$ with a definable set $Y^{\prime} \subseteq R^{\prime n}$ such that $Y=Y^{\prime} \cap R^{n}$. Then $Y^{\prime}$ is defined in $R^{\prime}$ by a formula $\phi(a, y)$ where $a \in R^{\prime m}$ and $\phi(x, y)$ is a formula in the language of $R$, $x=\left(x_{1}, \ldots, x_{m}\right), y=\left(y_{1}, \ldots, y_{n}\right)$. By $\omega$-saturation of $R$ we can take $b \in R^{m}$ such that $\operatorname{tp}(b \mid \emptyset)=\operatorname{tp}(a \mid \emptyset)$. Let $Z \subseteq R^{n}$ be defined in $R$ by $\phi(b, y)$. Then $Y \cap \mathcal{O}^{n} \subseteq \bigcup_{\epsilon} Z^{\epsilon}$, where $\epsilon$ ranges over all positive infinitesimals and

$$
Z^{\epsilon}:=\left\{y \in R^{n}: d(y, Z) \leq \epsilon\right\} .
$$

Otherwise there would be $y \in Y \cap \mathcal{O}^{n}$ such that $d(y, Z)>\mathfrak{m}$, so for some $\mathcal{O}$-box $P \subseteq R^{n}$, we would have $P \cap Y \neq \emptyset$ and $P \cap Z=\emptyset$, a contradiction with $\operatorname{tp}(b \mid \emptyset)=\operatorname{tp}(a \mid \emptyset)$.

It follows that st $Y \subseteq$ st $Z$. We claim that $\operatorname{int}($ st $Y \triangle$ st $Z)=\emptyset$. Otherwise, we can take a box $B \subseteq \mathbb{R}^{n}$ such that $B \subseteq$ st $Z \backslash$ st $Y$, so the $V$-box lemma yields an $\mathcal{O}$-box $P \subseteq Z$ such that $P \cap Y=\emptyset$, contradicting $\operatorname{tp}(b \mid \emptyset)=$ $\operatorname{tp}(a \mid \emptyset)$. 
THEOREM 4.4. For all $n$,

$$
\operatorname{Def}^{n}\left(\mathbb{R}_{\text {ind }}\right)=\left\{\text { st } X: X \subseteq R^{n} \text { is a trace }\right\} .
$$

Proof. By Lemma 4.1.

$$
\left\{\text { st } X: X \subseteq R^{n} \text { is a trace }\right\}=\operatorname{Def}^{n}\left(\mathbb{R}^{*}\right),
$$

for all $n$, and it is clear that $\operatorname{Def}^{n}\left(\mathbb{R}_{\text {ind }}\right) \subseteq \operatorname{Def}^{n}\left(\mathbb{R}^{*}\right)$. So let $X \subseteq R^{n}$ be a trace. By Lemma 4.3, we can take $Y \in \operatorname{Def}^{n}(R)$ such that int(st $X \triangle$ st $Y$ ) $=\emptyset$, hence, by Lemma 4.2 , $\operatorname{Def}^{n}\left(\mathbb{R}^{*}\right) \subseteq \operatorname{Def}^{n}\left(\mathbb{R}_{\text {ind }}\right)$.

Corollary 4.5. $\operatorname{Def}^{n}\left(\mathbb{R}_{\text {ind }}\right)=\left\{\operatorname{st} X: X \in \operatorname{Def}^{n}(R, \mathcal{O})\right\}$ for all $n$.

Proof. It is clear that $\left\{\right.$ st $\left.X: X \in \operatorname{Def}^{n}(R, \mathcal{O})\right\} \subseteq \operatorname{Def}^{n}\left(\mathbb{R}^{*}\right)$, so by Theorem 4.4. $\left\{\right.$ st $\left.X: X \in \operatorname{Def}^{n}(R, \mathcal{O})\right\} \subseteq \operatorname{Def}^{n}\left(\mathbb{R}_{\text {ind }}\right)$. To see that

$$
\operatorname{Def}^{n}\left(\mathbb{R}_{\text {ind }}\right) \subseteq\left\{\text { st } X: X \in \operatorname{Def}^{n}(R, \mathcal{O})\right\},
$$

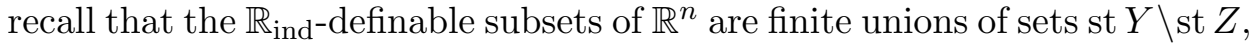
where $Y, Z \in \operatorname{Def}^{n}(R)$, and observe that

$$
\text { st } Y \backslash \text { st } Z=\operatorname{st}\{x \in Y: d(x, Z)>\mathfrak{m}\},
$$

and that $\mathfrak{m}$ is definable in the structure $(R, \mathcal{O})$.

\section{Open problems}

1. We showed that if cofinality $(\mathfrak{m})>2^{|\boldsymbol{k}|}$, then $(R, V) \models \Sigma$. Conversely, if $(R, V) \models \Sigma$, is there an elementary extension of $(R, V)$ satisfying this inequality?

2. Does an analogue of Corollary 4.5 hold under more general conditions, for example $(R, V) \models \Sigma$ ?

3. Let $R$ be an $\omega$-saturated elementary extension of the Lipshitz-Robinson structure. Are the definable sets of $\mathbb{R}_{\text {ind }}$ just the semialgebraic sets?

REMARK. An earlier version of this paper included a question by Lou van den Dries and Jonathan Kirby: Let $R$ be $\omega$-saturated and $V=\mathcal{O}$; is $\mathbb{R}_{\text {ind }}$ elementarily equivalent to a definable reduct of $R$ ?

However, a negative answer to this question follows from an observation by Tom Foster in [9]: Let $R$ be an $\omega$-saturated model of the real exponential field, and let $R^{\prime}$ be the $\left(+, \cdot,<, x^{c}\right)$-reduct of the expansion of $R$ by a function symbol $x^{c}$ for the $R$-definable function

$$
x^{c}: R^{>0} \rightarrow R^{>0}: x \mapsto \exp (c \log x),
$$

where $c \in R^{>\mathcal{O}}$. Then $R^{\prime}$ is o-minimal and power-bounded. On the other hand, the function

$$
(0, \infty) \rightarrow R^{>0}: x \mapsto(1+x / c)^{c}
$$


is definable in $R^{\prime}$, and the image of its graph under the residue map corresponding to $\mathcal{O}$ yields the graph of the exponential in $\mathbb{R}_{\text {ind }}$.

Acknowledgments. This paper contains some of the results in the author's PhD thesis. The author would like to thank her advisor Lou van den Dries for advice.

\section{References}

[1] Y. Baisalov et B. Poizat, Paires de structures o-minimales, J. Symbolic Logic 63 (1998), 570-578.

[2] B. S. Baizhanov, Expansion of an o-minimal model by unary convex predicates, in: Researches in Theory of Algebraic Systems, T. Nurmagambetov (ed.), Karaganda State Univ., 1995, 3-23.

[3] G. Cherlin and M. A. Dickmann, Real closed rings II: Model theory, Ann. Pure Appl. Logic 25 (1983), 213-231.

[4] L. van den Dries, O-minimal structures, in: Logic: from Foundations to Applications (Staffordshire, 1993), Oxford Sci. Publ., Oxford Univ. Press, New York, 1996, $137-185$.

[5] —, T-convexity and tame extensions II, J. Symbolic Logic 62 (1997), 14-34; Erratum, ibid. 63 (1998), 1597.

[6] —, Tame Topology and o-Minimal Structures, London Math. Soc. Lecture Note Ser. 248, Cambridge Univ. Press, 1998.

[7] L. van den Dries and A. H. Lewenberg, T-convexity and tame extensions, J. Symbolic Logic 60 (1995), 74-102.

[8] L. van den Dries and J. Maříková, Triangulation in o-minimal fields with standard part map, Fund. Math. 209 (2010), 133-155.

[9] T. Foster, The first order theory of raising to an infinite power, preprint.

[10] E. Hrushovski and Y. Peterzil, A question of van den Dries and a theorem of Lipshitz and Robinson; not everything is standard, J. Symbolic Logic 72 (2007), 119-122.

[11] E. Hrushovski, Y. Peterzil and A. Pillay, Groups, measures and the NIP, J. Amer. Math. Soc. 21 (2008), 563-596.

[12] L. Lipshitz and Z. Robinson, Overconvergent real closed quantifier elimination, Bull. London Math. Soc. 38 (2006), 897-906.

[13] D. Macpherson, D. Marker and C. Steinhorn, Weakly o-minimal structures and real closed fields, Trans. Amer. Math. Soc. 352 (2000), 5435-5483.

[14] J. Maříková, The structure on the real field generated by the standard part map on an o-minimal expansion of a real closed field, Israel J. Math. 171 (2009), 175-195.

[15] —, O-minimal residue fields of o-minimal fields, arXiv:1001.1575.

Jana Maříková

Department of Mathematics, WIU

476 Morgan Hall, 1 University Circle

Macomb, IL 61455, U.S.A.

E-mail: J-Marikova@wiu.edu 